\title{
Modification of p53 Immunoexpression Associated with Chemotherapy Regimens in Advanced and Refractory Ovarian Cancers
}

\author{
Takeshi Hirasawa $^{1}$, Masanori Yasuda ${ }^{2}$, Hironobu Maeda ${ }^{1}$, Toshinari Muramatsu ${ }^{1}$, \\ Tsuyoshi Miyamoto', Masaru Murakami', Takao Shinozuka ${ }^{1}$, Susumu Takekoshi ${ }^{2}$, \\ R. Yoshiyuki Osamura ${ }^{1}$ and Tsunehisa Makino ${ }^{1}$
}

Departments of ${ }^{1}$ Obstetrics and Gynecology and ${ }^{2}$ Pathology, School of Medicine, Tokai University, Bohseidai, Isehara, Kanagawa 259-1193, Japan

Received May 23, 2003; accepted September 24, 2003

For 34 advanced and refractory cases of epithelial ovarian cancer, we immunohistochemically analyzed modification of p53 expression in tissue from both primary and secondary surgeries, focusing on the relationship with chemotherapeutic effects caused by two different regimens: high-dose chemotherapy (HDC) and conventional chemotherapy (CONV). Following the primary surgery, 18 cases were treated with HDC and 16 cases with CONV. The p53 expression was more markedly decreased in the HDC-treated cases $\mathbf{( 7 8 \%}$ for the primary; $39 \%$ for the secondary), compared to that in the CONV-treated cases $(50 \%$ for the prima- ry; $43 \%$ for the secondary). Sixty-seven percent $(12 / 18)$ of the HDC-treated cases showed an obvious decrease in p53 expression, whereas $81 \%(13 / 16)$ of the CONV-treated cases showed no change in expression. In the comparison of these two groups, the survival ratio was higher in the 12 HDC-treated cases than that in the 13 CONV-treated cases $(p<0.05)$. In conclusion, as p53 expression in epithelial ovarian cancers is considerably influenced by chemotherapy regimen, it was suggested that the regimen yielding a distinct decrease in expression could yield a more favorable outcome.

Key words: p53, immunohistochemistry, high-dose chemotherapy, ovarian cancer

\section{Introduction}

Regarding epithelial ovarian cancers, no significant difference in the treatment outcome is recognized between CAP (cyclophosphamide+adriamycin+cisplatin) and cyclophosphamide+cisplatin $[5,15]$. The latter has been widely favored because of the lighter side effects for patients. Later, paclitaxel as an effective anti-cancer drug for ovarian cancer appeared, and the combination of paclitaxel+cisplatin+ cyclophosphamide has been proven to give a relatively good clinical outcome [13]. Recently, the mixture of paclitaxel+carboplatin has become the golden standard of chemotherapy for ovarian cancers due to its fewer side effects $[3,16]$. However, it is not fully satisfactory when the long-term prognosis results are analyzed.

Our department started high-dose chemotherapy

Correspondence to: Masanori Yasuda, M.D., Department of Pathology, School of Medicine, Tokai University, Bohseidai, Isehara, Kanagawa 259-1193, Japan. E-mail: m-yasuda@is.icc.u-tokai.ac.jp
(HDC) as a specific regimen for epithelial ovarian cancers 16 years ago. Compared to conventional chemotherapy $(\mathrm{CONV})$, the regimen of HDC is particularly characterized by the dose-dependent effect of platinum. We considered that an increased dose of platinum over the secondary threshold would be enforced by autologous stem cell support $[1,24]$. As a result, the clinical outcome has been considerably improved as well as the safety for the patients $[19,20]$. In ovarian cancers, oncogenes such as HER-2 [22], c-myc [29], and $k$-ras [4] were reported to be amplified, although the frequencies were not very high. Compared to these oncogenes, the abnormality of p53 expression is more frequently manifested in association with the morphological feature, clinicopathological state and prognosis not only in ovarian cancers, but also neoplasms of various organs [7, 11, 23, 2527]. Namely, the p53 expression appears at a high rate according to the advance of the stage [10]. Overexpression of $\mathrm{p} 53$ is believed to be one of the most reliable pathologic factors that influence the prognosis of ovarian cancers $[6,9$, $10]$. 
The present study focused on the retrospective analysis of the relationship between the modification of p53 expression and treatment effect or prognosis, associated with the different chemotherapy regimens, HDC and CONV. Our results suggested that p53 expression tended to be influenced more strongly by HDC than CONV.

\section{Patients and Methods}

\section{Case selection}

Of 226 ovarian cancers which were surgically treated at Tokai University Hospital during 1983 to 1995, 34 advanced and refractory epithelial cancers were chosen on the following conditions. a) The primary surgery was optimal or suboptimal. b) The secondary surgery after chemotherapy was performed to reduce or resect the recurrent tumors. The patients were aged from 32 to 74 years old (mean 54). The stage according to classification of International Federation of Gynecology and Obstetrics (FIGO), histology and residual tumor size are shown in Table 1. The residual tumor size at the primary surgery was divided into two groups: minimal and $>1 \mathrm{~cm}$. There was no significant difference in the survival ratio between the HDC-treated cases and CONV-treated cases (Fig. 1).

Following the primary surgery, 18 patients were treated with HDC (cisplatin $300 \mathrm{mg} / \mathrm{m}^{2}$ and cyclophosphamide $3000 \mathrm{mg} / \mathrm{m}^{2}$ ) and 16 with CONV (cisplatin $50-100 \mathrm{mg} / \mathrm{m}^{2}$, adriamycyn $50 \mathrm{mg} / \mathrm{m}^{2}$, cyclophosphamide $500 \mathrm{mg} / \mathrm{m}^{2}$ or carboplatin $1500 \mathrm{mg} / \mathrm{m}^{2}$ ). As for the neoadjuvant chemotherapy, only platinum was administered intraperitoneally or intravenously to 19 patients, mainly because of the massive ascites storage with cancer cell dissemination.
Table 1. Ovarian epithelial cancers studied

\begin{tabular}{cc}
\hline & No. of case \\
\hline FIGO stage & \\
Ic/II & 4 \\
III & 19 \\
IV & 11 \\
Histological type & \\
Serous & 24 \\
Non-serous & 10 \\
Residual tumor & \\
minimal & 16 \\
$<1$ cm & 18 \\
\hline FIGO: International & Federation of Gynecology \\
and Obstetrics. &
\end{tabular}

Two tissue samples for each case were obtained from the primary and secondary surgery specimens, respectively. The secondary surgery specimens included lymph nodes, intestinal tract, liver, spleen, and peritoneum.

\section{Immunohistochemical staining of p53}

The surgical specimens of ovarian epithelial cancer used were routinely formalin-fixed and paraffin-embedded. The representative 68 sections in $4 \mu \mathrm{m}$ thick were deparaffinized and processed with heat antigen retrieval at $90^{\circ} \mathrm{C}$ for $5 \mathrm{~min}$ in $0.01 \mathrm{M}$ phosphate buffered saline (PBS), $\mathrm{pH} 7.2$, using a microwave. The labeled streptoavidin-biotin method was applied. The primary antibody (clone DO7, Novocastra Laboratories Tyne, UK) was incubated at a dilution of 1:50. Sections were counterstained with $5 \%$ methyl green. As a

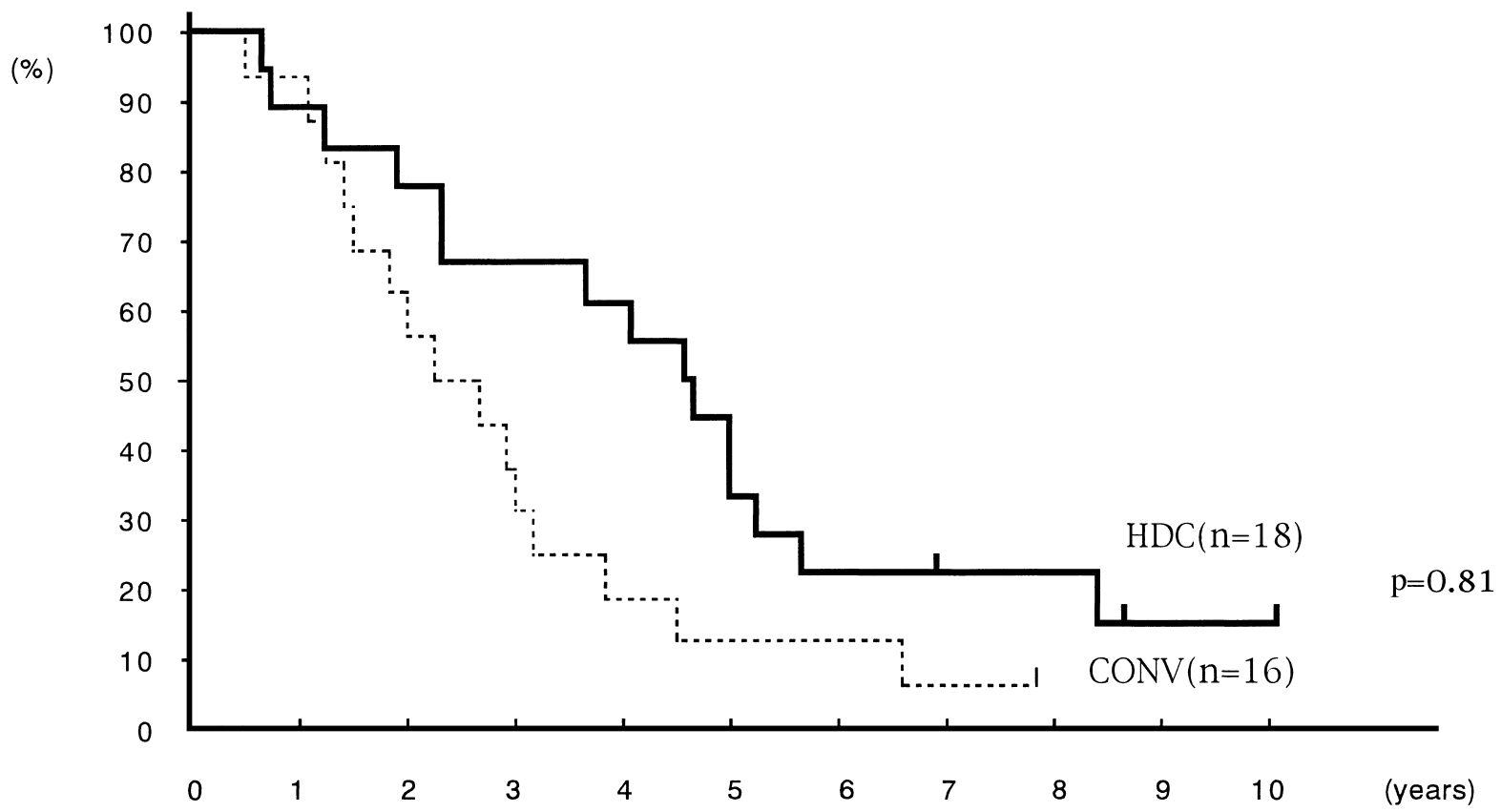

Fig. 1. The survival curve of CONV and HDC in the 34 cases (Kaplan-Meier Method). HDC, high-dose chemotherapy; CONV, conventional chemotherapy. 
positive control, known colon carcinomas were used. For the negative control study, sections were incubated with a non-immunized serum instead of the primary antibody. A labeling index, which was defined as positive cells/1,000 tumor cells, was categorized into five groups: less than 5\%, negative; +, 5-25\%; 2+, 25-50\%; 3+, 50-75\%; 4+, 75\%<.

\section{Statistical analysis}

The data were analyzed by means of the $\chi^{2}$ or Fisher's exact test. $\mathrm{p}<0.05$ was regarded as significant. The KaplanMeier curve was utilized to evaluate the survival ratio.

\section{Results}

The alterations in the positive ratios of p53 expression from the primary to the secondary surgery are shown in Table 1. The overall positive ratios in the primary and secondary were $65 \%(22 / 34)$ and $41 \%(14 / 34)$, respectively. For the 18 HDC-treated cases, the positive ratio was decreased from the primary to the secondary surgery: $78 \%$ $(14 / 18)$ to $39 \%(7 / 18)(p<0.02)$, as shown in Table 2 . In contrary, no remarkable decrease was noted in the $16 \mathrm{CONV}$ treated cases: $50 \%(8 / 16)$ to $43 \%(7 / 16)$, as shown in Table 2. According to the follow-up period between the primary to the secondary surgery, the 34 cases were divided into 2 groups: 16 cases, $<1$ year, and 18 cases, $>1$ year. The decrease of positive ratios was more evident in the latter: from $72 \%(13 / 18)$ to $33 \%(6 / 18)(\mathrm{p}<0.02)$ than in the former: from $56 \%(9 / 16)$ to $50 \%(8 / 16)$, as shown in Table 3 .

The modification of p53 expression after HDC and CONV was semi-quantitatively analyzed, as shown in Table
4. Sixty-seven percent (12/18) of the HDC-treated cases exhibited a decrease in the positive ratio, however, in $81 \%$ $(13 / 16)$ of the CONV-treated cases no obvious change was noted. Among 7 of 12 HDC-treated cases with varying decrease of p53 expression, no positive reaction was observed in the secondary surgery. One case in each group showed a p53 expression increase. A difference in modification between the two groups of $\mathrm{p}<0.02$ was regarded as statistically significant.

In the comparison of the survival ratio for the 12 HDCtreated cases showing a decrease in p53 expression and that for the $13 \mathrm{CONV}$-treated cases showing no change, as demonstrated in Fig. 2, a more favorable outcome was shown in the HDC-treated cases $(\mathrm{p}<0.05)$.

\section{Discussion}

The main reasons for focusing on p53 expression are that this protein can be easily applied for evaluating the biological behavior of various kinds of neoplasms [7, 11, 23, 25-27], including ovarian cancers [6, 9, 10], and that many reports have mentioned that the overexpression of p53 is closely associated with an unfavorable outcome. For ovarian cancers with a high grade and/or in an advanced stage, mutant p53 protein accumulation is not a significant prognosisrelated factor that reflects an increased risk for relapse and death for tumors [10]. Kiyokawa et al. [8] reported that no significant correlation was found between the aberrant expression of p53 and stage or histologic type, and that there was a concordant expression level of p53 in primary and matched metastatic lesions. Silvestrini et al. [21] also men-

Table 2. p53 expression: Comparison of positive ratios in primary and secondary surgery

\begin{tabular}{|c|c|c|c|c|}
\hline & No. of cases & Primary surgery & Secondary surgery & $\mathrm{p}$ value \\
\hline & & p53 expression & p53 expression & \\
\hline & & Positive ratio & Positive ratio & \\
\hline & & $\%(n)$ & $\%(n)$ & \\
\hline Total cases & 34 & $65(22)$ & $41(14)$ & $\mathrm{p}<0.05$ \\
\hline HDC & 18 & $78(14)$ & $39(7)$ & $\mathrm{p}<0.02$ \\
\hline CONV & 16 & $50(8)$ & $43(7)$ & NS \\
\hline
\end{tabular}

HDC, high-dose chemotherapy; CONV, conventional chemotherapy.

Table 3. p53 expression: Comparison of positive ratios in primary and secondary surgery

\begin{tabular}{ccccc}
\hline & No. of cases & Primary surgery & Secondary surgery & $\mathrm{p}$ value \\
\cline { 3 - 3 } & & $\mathrm{p} 53$ expression & $\mathrm{p} 53$ expression \\
\cline { 3 - 4 } & & $\begin{array}{c}\text { Positive ratio } \\
\%(\mathrm{n})\end{array}$ & $\begin{array}{c}\text { Positive ratio } \\
\%(\mathrm{n})\end{array}$ \\
\hline $\begin{array}{c}\text { Interval } \\
<1 \text { year }\end{array}$ & 16 & $56(9)$ & $50(8)$ & $\mathrm{NS}$ \\
$\geq 1$ year & 18 & $72(13)$ & $33(6)$ & $\mathrm{p}<0.02$ \\
\hline
\end{tabular}

Interval: period during primary to secondary surgery. 
Table 4. Modification of $p 53$ expression from primary to secondary surgery

\begin{tabular}{lcc}
\hline & $\begin{array}{c}\text { HDC-treated cases (18) } \\
\%(\mathrm{n})\end{array}$ & $\begin{array}{c}\text { CONV-treated cases (16) } \\
\%(\mathrm{n})\end{array}$ \\
\hline Decrease & ${ }^{\mathrm{a}} 67(12)$ & ${ }^{\mathrm{b}} 13(2)$ \\
No change & $\mathrm{c} 28(5)$ & $\mathrm{d} 81(13)$ \\
Increase & $5(1)$ & $6(1)$ \\
\hline
\end{tabular}

decrease: + to $-[6$ cases (CONV: 1 cases, HDC: 5 cases) $], 2+$ to (HDC: 1 case), $3+$ to - (HDC: 1 case), $3+$ to $2+$ (CONV: 1 case), $4+$ to $3+$ (HDC: 5 cases)

no change: - to $-[12$ cases (CONV: 8 cases, HDC: 4 cases)], + to + (CONP: 2 cases), $4+$ to $4+$ [ 4 cases (CONV: 3 cases, HDC: 1 case)] increase: $2+$ to $3+$ (HDC: 1 case), $3+$ to $4+$ (CONV: 1 case)

$\mathrm{p}=0.02$ : Fisher's exact test [between $(\mathrm{a}, \mathrm{d})$ and $(\mathrm{b}, \mathrm{c})]$

tioned that alterations in the accumulated p53 protein are not correlated with clinical response or survival after cisplatin treatment. In our experience so far, regarding refractory ovarian epithelial cancers, immunohistochemical p53 expression profiles seem to be less changeable, however, a distinct decrease of p53 expression has been occasionally recognized after chemotherapy.

In the present study, we attempted to clarify the modification of p53 expression from the viewpoint of the association with therapeutic effects caused by two different chemotherapy regimens. In the 18 HDC-treated cases, the positive ratios were drastically decreased from $78 \%$ for the primary surgery to $39 \%$ for the secondary surgery $(\mathrm{p}<0.02)$ (Table 2). On the contrary, no significant decrease was noted in the CONV-treated cases. Sixty-seven percent (12/18) of the HDC-treated cases and $37 \%(6 / 16)$ of the CONV-treated cases had a follow-up period of more than 1 year, respectively. Thus, the chemotherapeutic effects seem to be reflected in the elongation of the follow-up period between the primary and secondary surgeries (Table 3 ), and are considered to be closely associated with a decrease in p53 expression.

The semi-quantitative analysis with the modification of the p53 expression from the primary to the secondary surgery provides a reasonable explanation for the above interpretation (Table 4). In fact, a decrease in the grade of the positive extent was noted in $67 \%$ of the HDC-treated cases, but in only $13 \%$ of the CONV-treated cases. In the majority (81\%) of the CONV-treated cases, no significant difference in p53 expression was noted between the primary and secondary surgeries. The difference in the survival ratios was found considerably favorable with the 12 HDC-treated cases showing a decrease in p53 expression, compared to the 13 CONV-treated cases showing no change $(\mathrm{p}<0.05)$ (Fig. 2). In the analysis of the relationship between p53 expression and chemosensitivity [17, 18, 28], Righetti et al. [17] mentioned that a significant correlation was found between p53 accumulation, type of mutation, and pathological response to cisplatin-based therapy. Buttitta et al. [2] also reported that ovarian cancers showing p53 aberrations are significantly less sensitive to chemotherapy and more aggressive than those with functional p53. In addition, they observed that patients with p53 mutations had a significantly shorter progression-free survival than those with p53-negative tumors [2]. From our results, it is speculated that immunohistochemical p53 expression could be more susceptible to decrease by HDC than CONV, and that this phenomenon may be related to the difference in prognosis between HDCtreated cases and CONV-treated cases. Using the cell lines

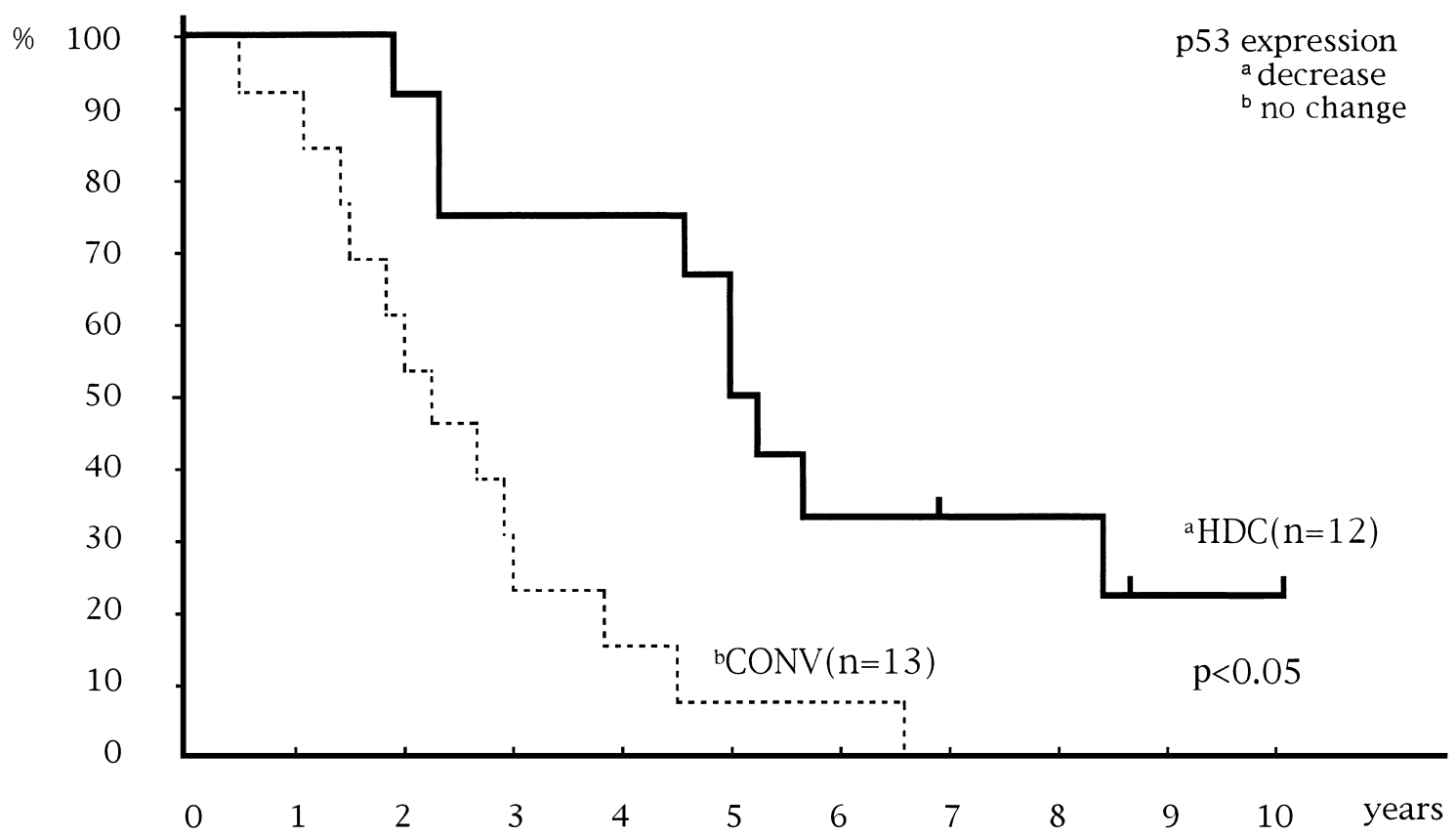

Fig. 2. Comparison of survival ratios of ${ }^{a} \mathrm{HDC}$-treated cases and ${ }^{\mathrm{b}} \mathrm{CONV}$-treated cases (Kaplan-Meier Method). HDC, high-dose chemotherapy; CONV, conventional chemotherapy. 

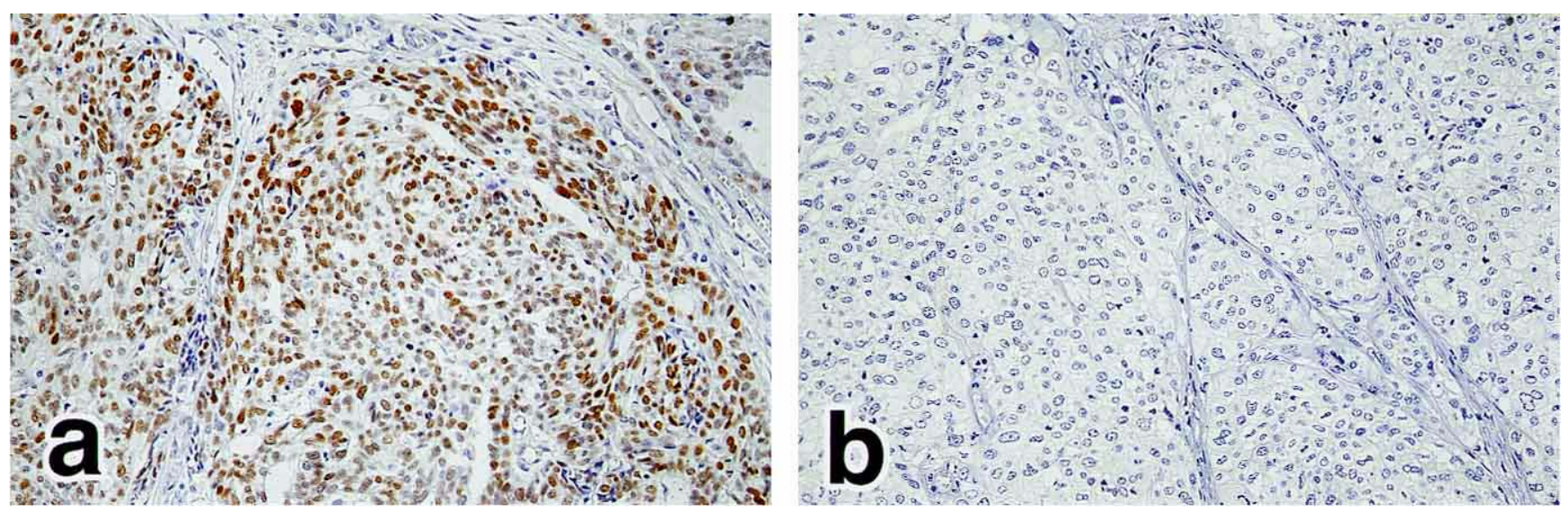

Fig. 3. HDC-treated case with endometrioid adenocarcinoma. Intense p53 expression before HDC (a) completely disappeared after HDC (b). Original magnification $\times 50$
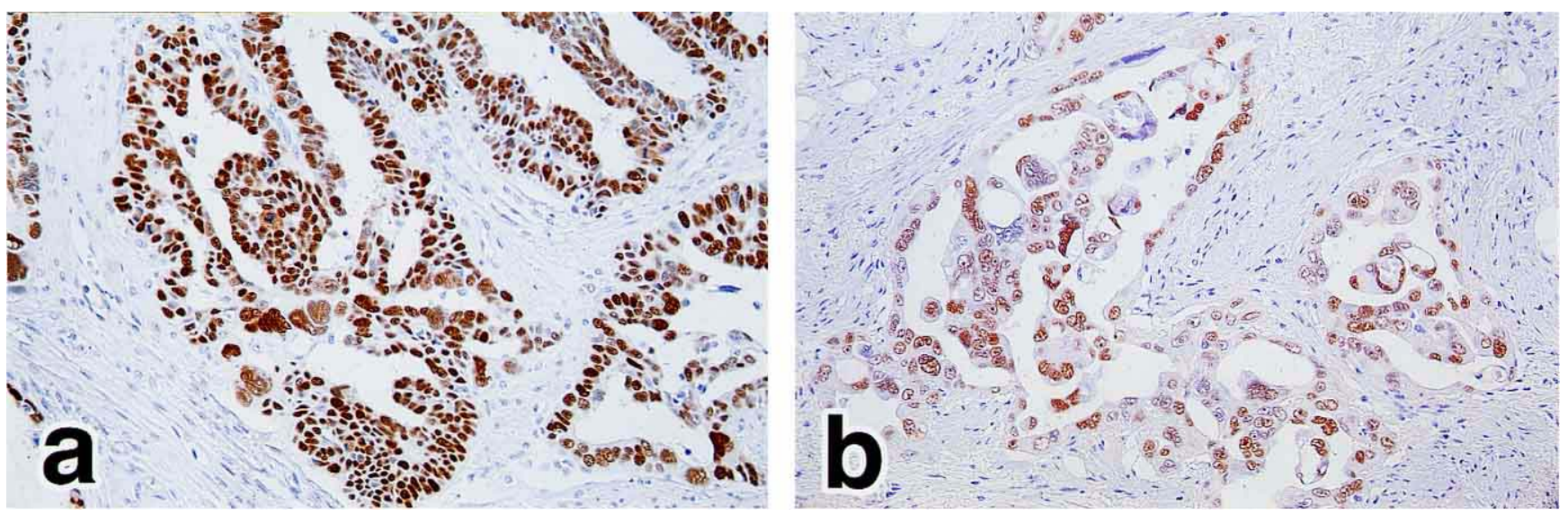

Fig. 4. CONV-treated case with serous adenocarcinoma. No significant change in $\mathrm{p} 53$ expression was noted between before (a) and after CONV

(b). Original magnification $\times 50$

of ovarian cancer in a variable p53 status, it has been demonstrated that occurrence of apoptosis depends on the drug and the drug dose used [14]. The effect of high cisplatin concentration for a cell line exhibiting mutant p53 could be the same as that of the low cisplatin concentration for a cell line exhibiting wild p53. Therefore, irrespective of the abnormal p53 expression in the primary surgical specimen, the expression is expected to be downregulated by appropriate chemotherapy for the recurrent or residual tumors. It seems that, on this point, p53 overexpression may not be necessarily an indicator of the poor clinical outcome. Although, there is a statement that a significant dose-response effect of cisplatin is not seen in patients with p53 positive ovarian tumors, unlike those with p53 negative ovarian tumors [12].

In the present study, the number of cases used was not sufficient to perform multivariate analyses for the relationship between p53 expression and histologic type, stage, effect of neo-adjuvant therapy, and residual tumor size. Nevertheless, we stress that the HDC-treated cases tended to exhibit a decreased immunohistochemical expression of p53 in comparison to the CONV-treated cases. Focusing on the appropriate selection of chemotherapy regimens for ovarian cancer patients, we are trying to investigate the optimal conditions for inducing apoptosis, using human ovarian cancer cell lines with or without p53 mutations. In parallel to this project, a prospective randomized trial for epithelial ovarian cancers applying HDC and CONV is being attempted [19].

\section{Acknowledgments}

We dedicate this paper to the memory of the late Dr. Keiichi Watanabe, Professor of Department of Pathology, School of Medicine, Tokai University, for his enthusiastic instruction on the basis of immunohistochemistry and valuable suggestions on the application of this technique.

\section{References}

1. Aghajanian, C., Fennelly, D., Shapiro, F., Waltzman, R., Almadrones, L., O'Flaherty, C., O'Conner, K., Venkatraman, E., Barakat, R., Curtin, J., Brown, C., Reich, L., Wuest, D., Norton, 
L., Hoskins, W. and Spriggs, D. R. (1998) Phase II study of "dose-dense" high-dose chemotherapy treatment with peripheralblood progenitor-cell support as primary treatment for patients with advanced ovarian cancer. J. Clin. Oncol. 16; 1852-1860.

2. Buttitta, F., Marchetti, A., Gadducci, A., Pellegrini, S., Morganti, M., Carnicelli, V., Cosio, S., Gagetti, O., Genazzani, A. R. and Bevilacqua, G. (1997) p53 alterations are predictive of chemoresistance and aggressiveness in ovarian carcinomas: a molecular and immunohistochemical study. Br. J. Cancer 75; 230-235.

3. du Bois, A., Lueck, H. J., Meier, W., Moebus, V., Costa, S. D., Bauknecht, T., Richter, B., Warm, M., Schroeder, W., Olbricht, S., Nitz, U. and Jackisch, C. (1999) Cisplatin/paclitaxel vs carboplatin/paclitaxel in ovarian cancer: Update of an Arbeitgemeinschaft Gynecologische Oncologie (AGO) study group trial. Proc. Am. Soc. Clin. Oncol. 18; 356a (No. 1374).

4. Filmus, J. E. and Buick, R. N. (1985) Stability of c-K-ras amplification during progression in a patient with adenocarcinoma of the ovary. Cancer Res. 45; 4468-4472.

5. Gruppo Interegionale Cooperativo Oncologico Ginecologia (1987) Randomized comparison of cisplatin with cyclophosphamide/cisplatin and with cyclophophamide/doxorubicin/cisplatin in advanced ovarian cancer. Lancet 2; 353-359.

6. Herod, J. J., Elipoulos, A. G., Warwick, J., Niedobitek, G., Young, L. S. and Kerr, D. J. (1996) The prognostic significance of Bcl-2 and p53 expression in ovarian carcinoma. Cancer Res $56 ; 2178-2184$.

7. Kashima, K., Yokoyama, S., Nakayama, I. and Noguchi, S. (1991) Immunohistochemical study on expression of c-myc, p53, c-erbB-2 and epithelial growth factor receptor in human thyroid tumors. Acta Histochem. Cytochem. 24; 563-570.

8. Kiyokawa, T. (1994) Alteration of p53 in ovarian cancer: its occurrence and maintenance in tumor progression. Int. J. Gynecol. Pathol. 13; 311-318.

9. Klemi, P. J., Pylkkanen, L., Kiilholma, P., Kurvinen, K. and Joensuu, H. (1995) p53 protein detected by immunohistochemistry as a prognostic factor in patients with epithelial ovarian carcinoma. Cancer 76; 1201-1208.

10. Levesque, M. A., Katsaros, D., Yu, H., Zola, P., Sismondi, P., Giardina, G. and Diamandis, E. P. (1995) Mutant p53 protein overexpression is associated with poor outcome in patients with well or moderately differentiated ovarian cancer. Cancer 75; $1327-1338$.

11. Lipponen, P. K. (1993) Over-expression of p53 nuclear oncoprotein in transitional-cell bladder cancer and its prognostic value. Int. J. Cancer 53; 365-370.

12. Marx, D., Meden, H., Ziemek, T., Lenthe, T., Kuhn, W. and Schauer, A. (1998) Expression of $p 53$ tumour suppressor gene as a prognostic marker in platinum-treated patients with ovarian cancer. Eur. J. Cancer 34; 845-850.

13. McGuire, W. P., Hoskins, W. J., Brady, M. F., Kucera, P. R., Partridge, E. E., Look, K. Y., Clarke-Pearson, D. L. and Davidson, M. (1996) Cyclophophamide and cisplatin compared with paclitaxel and cisplatin in patients with stage III and IV ovarian cancer. N. Engl. J. Med. 334; 1-6.

14. Merlin, T., Brandner, G. and Hess, R. D. (1998) Cell cycle arrest in ovarian cancer cell lines dose not depend on p53 status upon treatment with cytostatic drugs. Int. J. Oncol. 13; 1007-1016.

15. Omura, G. A., Bundy, B. N., Berek, J. S., Curry, S., Delgado, G. and Mortel, R. (1989) Randomized trial of cyclophophamide plus cisplatin with or without doxorubicin in ovarian carcinoma: a Gynecologic Oncology Group Study. J. Clin. Oncol. 7; 457-465.
16. Ozols, R. F., Bundy, B. N., Fowler, J., Clarke-Pearson, D., Mannel, R., Hartenbach, E. M. and Baergen, R. (1999) Randomized phase III study of cisplatin/paclitaxel vs carboplatin/paclitaxel in optimal stage III ovarian cancer. A gynecologic oncology group trial (GOG158). Proc. Am. Soc. Clin. Oncol. 18; 356a (No. 1373).

17. Righetti, S. C., Della Torre, G., Pilotti, S., Menard, S., Ottone, F., Colnaghi, M. I., Pierotti, M. A., Lavarino, C., Cornarotti, M., Oriana, S., Bohm, S., Bresciani, G. L., Spatti, G. and Zunino, F. (1996) A comparative study of $p 53$ gene mutations, protein accumulation, and response to cisplatin-based chemotherapy in advanced ovarian carcinoma. Cancer Res. 56; 689-693.

18. Shelling, A. N. (1997) Role of p53 in drug resistance in ovarian cancer. Lancet 349; 744-745.

19. Shinozuka, T., Miyamoto, T., Muramatsu, T., Hirasawa, T., Murakami, M., Makino, T. and Tanaka, Y. (1999) High dose chemotherapy with autologous stem cell support in the treatment of patients with ovarian carcinoma. Long term results for 105 patients. Cancer $85 ; 1555-1564$.

20. Shinozuka, T., Muramatsu, T., Miyamoto, T., Hirasawa, T., Murakami, M., Makino, T., Sadahiro, S., Tanaka, Y. and Yasuda, M. (1999) Long-term results and prognostic analysis in advanced and recurrent/refactory epithelial ovarian cancer treated by highdose cyclophophamide, adriamycin, and cisplatin with autologous bone marrow transplantation. Int. J. Clin. Oncol. 4; 273279.

21. Silvestrini, R., Daidone, M. G., Veneroni, S., Benini, E., Scarfone, G., Zanaboni, F., Villa, A., Presti, M., Danese, S. and Bolis, G. (1998) The clinical predictivity of biomarkers of stage III-IV epithelial ovarian cancer in a prospective randomized treatment protocol. Cancer 82; 159-167.

22. Slamon, D. J., Godolphin, W., Jones, L. A., Holt, J. A., Wong, S. G., Keith, D. E., Levin, W. J., Stuart, S. G., Ullrich, A. and Press, M. F. (1989) Studies of the HER-2/neu proto-oncogene in human breast and ovarian cancer. Science 244; 707-712.

23. Starzynska, T., Bromley, M., Ghosh, A. and Stern, P. L. (1992) Prognostic significance of $\mathrm{p} 53$ overexpression in gastric and colorectal carcinoma. Br. J. Cancer 66; 558-562.

24. Thigpen, J. T. (1997) Dose-intensity in ovarian carcinoma; hold, enough? J. Clin. Oncol. 15; 1291-1293.

25. Thor, A. D., Moore, D. H. II, Edgerton, S. M., Kawasaki, E. S., Reihsaus, E., Lynch, H. T., Marcus, J. N., Schwartz, L., Chen, L. C., Mayall, B. H. and Smith, H. S. (1992) Accumulation of p53 tumor suppressor gene protein: an independent marker of prognosis in breast cancers. J. Natl. Cancer Inst. 84; 845-855.

26. Visakorpi, T., Kallioniemi, O-P., Heillinen, A., Koivula, T. and Isola, J. (1992) Small subgroup of aggressive, highly proliferative prostatic carcinomas defined by 553 accumulation. J. Natl. Cancer Inst. 11; 883-887.

27. Wada, R., Miwa, H., Abe, H., Kuwabara, N., Suda, K., Terai, T., Kondo, K. and Matsukawa, M. (1993) The possible association between expression of p53 and development in depressed type colorectal carcinoma. Acta Histochem. Cytochem. 26; 21-25.

28. Wahl, A. F., Donaldson, K. L., Fairchild, C., Lee, F. Y., Foster, S. A., Demers, G. W. and Galloway, D. A. (1996) Loss of normal p53 function confers sensitization to Taxol by increasing G2/M arrest and apoptosis. Nat. Med. 2; 72-79.

29. Zhou, D. J., Gonzalez-Cadavid, N., Ahuja, H., Battifora, H., Moore, G. E. and Cline, M. J. (1988) A unique pattern of protooncogene abnormalities in ovarian adenocarcinomas. Cancer 62; 1573-1576. 